and the cone which touches the planes $B O C, C O A, A O B$ and the coordinate planes is

$$
\sqrt{l_{1} l_{2} l_{3} x}+\sqrt{m_{1} m_{2} m_{3} y}+\sqrt{n_{1} n_{2} n_{3} z}=0 .
$$

Substituting for $l_{1} l_{2} l_{3}, m_{1} m_{2} m_{3}$, and $n_{1} n_{2} n_{3}$, we obtain the equations of these cones.

R. J. T. BELL.

\title{
A Method of obtaining Examples on the Multiplication of Determinants.
}

In the ordinary text-books on Algebra there is a lack of suitable examples on Multiplication of Determinants. Most of the examples that are given are particular cases of the theorem

in which

$$
D \triangle=D^{n}
$$

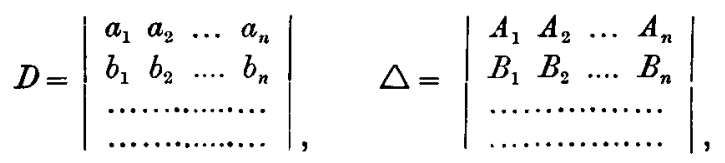

where $A_{1}, A_{2}, \ldots, B_{1}, \ldots$, are the co-factors of $a_{1}, a_{2}, \ldots, b_{1} \ldots$, in $D$.

If the determinant $D$ is chosen at random, in most cases the second determinant $\triangle$ will be too complicated. It is easy, however, to choose $D$ so that factors can be taken out of $\triangle$; and thus a sufficiently simple second determinant is obtained.

For example, let

$$
D=\left|\begin{array}{ccc}
b & a & a \\
a & b & a \\
a & a & b
\end{array}\right|=(2 a+b)(a-b)^{2} .
$$

Then

$$
\triangle=\left|\begin{array}{ccc}
b^{2}-a^{2} & a^{2}-a b & a^{2}-a b \\
a^{2}-a b & b^{2}-a^{2} & a^{2}-a b \\
a^{2}-a b & a^{2}-a b & b^{2}-a^{2}
\end{array}\right| .
$$

Let the factor $b-a$ be taken out of each row of $\triangle$. Then, multiplying the determinant so obtained by $D$, we have

$$
\begin{aligned}
\left|\begin{array}{lll}
b & a & a \\
a & b & a \\
a & a & b
\end{array}\right|\left|\begin{array}{rrr}
a+b & -a & -a \\
-a & a+b & -a \\
-a & -a & a+b
\end{array}\right| & =\left|\begin{array}{ccc}
b^{2}+b a-2 a^{2} & 0 & 0 \\
0 & b^{2}+b a-2 a^{2} & 0 \\
0 & 0 & b^{2}+b a-2 a^{2}
\end{array}\right| \\
& =(b-a)^{3}(b+2 a)^{3} .
\end{aligned}
$$


GEOMETRICAL PROOF OF TAN A +TAN B + TAN C= TAN A TAN B TAN C.

Similarly, starting from the first of the two following determinants, we obtain

$$
\left|\begin{array}{llll}
a & b & b & b \\
a & b & a & a \\
b & b & a & b \\
a & a & a & b
\end{array}\right|\left|\begin{array}{rrrr}
a & -a & b & -a \\
-b & b & -b & a \\
b & -a & a & -a \\
-b & a & -b & -b
\end{array}\right|=(a-b)^{8} .
$$

In this example the two determinants multiplied are really identical.

Thomas M. MacRobert.
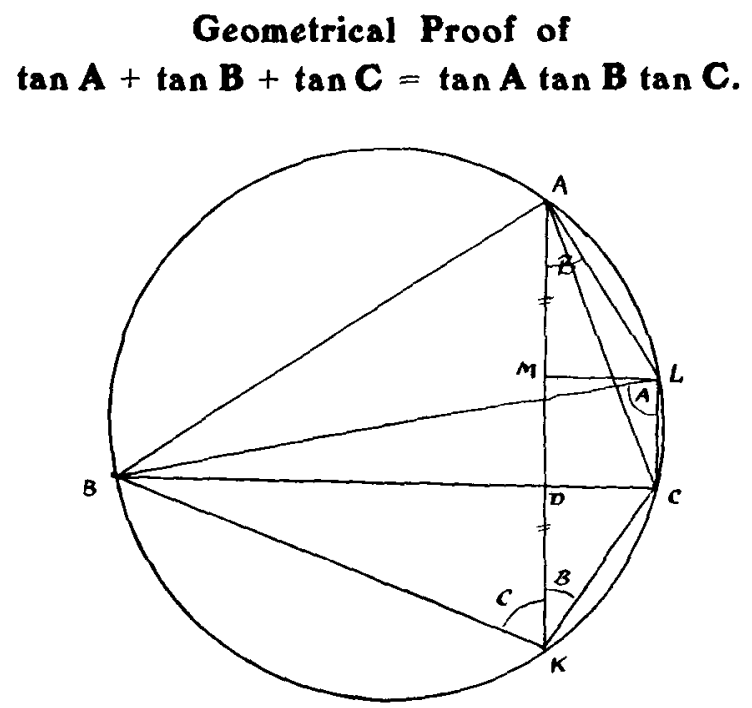

Let $A B C$ be the $\triangle, A D \perp$ to $B C$ produced to meet circumcircle in $K, B L$ a diameter of circumcircle, $L M \perp$ to $A K$. Let $B K, K C$, $C L$ and $L A$ be joined.

$$
\tan A=\frac{a}{C L}, \quad \tan B=\frac{D C}{D K}, \quad \tan C=\frac{D B}{D K} ;
$$

$\therefore \tan A+\tan B+\tan C=\frac{a}{C L}+\frac{a}{D K}=\frac{a(D K+C L)}{C L . D K}$

$$
\tan A \cdot \tan B \cdot \tan C=\frac{a}{C L} \times \frac{D C}{D K} \times \frac{D B}{D K}=\frac{a \cdot A D}{C L \cdot D K},
$$

since

$$
A D . D K=B D . D C \text {. }
$$

